\title{
Beyond starvation: An update on the autophagic machinery and its functions
}

\author{
Tsuyoshi Kawabata $^{1}$ and Tamotsu Yoshimori ${ }^{1,2}$ \\ ${ }^{1}$ Department of Genetics, Graduate School of Medicine, ${ }^{2}$ Laboratory of Intracellular \\ Membrane Dynamics, Graduate school of Frontier Biosciences, Osaka University, 2-2 \\ Yamadaoka, Suita, Osaka 565-0871, Japan
}

\begin{abstract}
Autophagy was originally identified as a cytoprotective system that provides emergency backup energy and basic building blocks under starvation condition by digesting self components. Recent advances in the field unveiled that this system also protects cells against multiple types of stress, as well as invasion by pathogens. Consistent with these findings, autophagy has been redefined as a safeguard system that plays a vital role in human pathology, and this realization has led to exponential progress in autophagy research. In this review, we introduce the basic mechanisms of canonical autophagy and also discuss selective autophagy, a set of pathways that target specific cellular components for digestion; in particular, we focus on lysophagy, a recently identified mechanism required for lysosomal homeostasis.
\end{abstract}

\section{Introduction}

Starvation is unquestionably one of the most lethal threats for organisms living in natural environments, where nutrient-rich conditions are uncommon; consequently, most organisms are forced to survive under recurrent starvation. To obtain the minimal levels of energy and building blocks required for survival, organisms had to develop mechanisms for digesting their own components. One such mechanism is autophagy (derived from Greek words meaning 'self-eating'), which is now recognized as a key cellular system for surviving emergency conditions such as starvation. In contrast to proteasome-dependent protein degradation, which specifically breaks down targets labeled with polyubiquitin chains, autophagy acts on a larger scale, degrading cytoplasmic components engulfed by an organelle (the double-membrane autophagosome) that ranges in size from 0.5 to $1.5 \mu \mathrm{m}[1]$. Several decades ago, C. de Duve identified the lysosome, an organelle with the high density of acidic hydrolases and low $\mathrm{pH}$ required for digestion; since that time, autophagy has been extensively investigated[2]. The most striking advance in the field was achieved in the early 1990's by Y. Ohsumi's group. Using elegant methods to quantify autophagic activity in yeast, they identified an essential core set of genes required for autophagy, some of which overlapped with genes independently identified by D. Klionsky's and H.D. Wolf's groups [3-5]. After identification of these $A T G$ (Autophagy) genes, mechanistic studies of autophagy expanded enormously, due not only to a series of elegant findings highlighting the highly systematic nature of autophagic regulation, but also to a redefinition of autophagy as a system that safeguards against a broad range of diseases and threats, including neurodegenerative disorders, cancer, arterial sclerosis, type 2 diabetes, heart failure, Crohn's disease, and bacterial infections $[6]$.

In this review, we first describe the basic mechanisms of autophagy in response to starvation stress, and then summarize recent advances in the field that illustrate the role of 
autophagy in digesting intracellular "rogues" to prevent life-threatening diseases. The history and precise regulatory mechanisms of autophagy have been elegantly presented elsewhere[2]. Therefore, here we emphasize the role of mammalian autophagy and introduce a recently identified form of selective autophagy, lysophagy. This pathway shares essential components required for conventional autophagy, but is fundamentally distinct from other forms of selective autophagy because it is uniquely responsible for regulating the process of autophagy itself. We focus on macroautophagy (called simply 'autophagy' in this review), which is mediated by sequestration of cytoplasmic components by an isolation membrane, rather than chaperone-mediated autophagy (CMA), which is mediated by direct lysosomal targeting of proteins containing a specific consensus sequence via LAMP2A-Hsc70 on the lysosomal membrane (reviewed by A. M. Cuervo et al. in detail [7, 8]).

\section{The nutrient-sensing machinery regulates autophagy}

Obviously, autophagy should be induced in response to starvation. After identification of ATG1 (yeast homolog of ULK1), which plays a central role in activation of downstream component of autophagy, negative regulation of the ULK1 complex by mTOR under nutrient-rich conditions has been considered to be the principal axis of regulation of mammalian autophagy. Diligent work by D. Sabatini's group revealed that the recognition of "hunger" in the cells is performed, at least in part, on lysosomes[9]_(summarized in Figure 1). mTOR is a master regulator that turns on cellular proliferation by phosphorylating downstream kinases such as S6 kinase. A subunit of v-ATPase senses amino-acid levels within the lysosome; when these levels are sufficiently high, Rag GTPases are activated and bind to mTORC1, thereby maintaining mTOR in an active state [10, 11]. This active form of mTORC1 constitutively phosphorylates ULK1 on Ser757, as well as the ULK complex component ATG13, leading to inactivation of ULK [12-15]. Upon starvation, amino acid depletion causes dissociation of mTORC1 from the lysosome, followed by a decrease in phosphorylation of ULK1 and ATG13 and activation of autophagy. Simultaneously, starvation promotes dephosphorylation mediated by the PP2A complex [16]; consistent with this, the phosphatase inhibitor okadaic acid inhibits autophagy [17]. By contrast, AMPK positively regulates autophagy by phosphorylating ULK1 on Ser317 and Ser777, as well as inhibiting mTOR [18, 19]. The yeast ATG1 complex is regulated by dissociation of phosphorylated Atg13 from Atg1 [20], whereas mammalian ULK1 binds ATG13 constitutively [12].

In addition to amino acids, autophagy is also regulated by glucose levels. Soon after autophagy was identified in rat liver 30 years ago, it became clear that glucagon induces and insulin suppresses autophagy [21]. Recent studies show that glucagon may regulate autophagy through CREB and mTOR, as indicated by their tight association [22-25]. These facts strongly suggest that mTOR is a central upstream regulator of autophagy, but also indicate that cells have mTOR-independent modes of autophagy regulation [26-28]. Additionally, mTOR and CREB also transcriptionally regulate autophagy via TFEB, a transcriptional activator of autophagy/lysosomal digestion [22, 29, 30].

\section{Machinery required for autophagosome formation}


Because autophagy is a spatiotemporally dynamic process that engulfs a part of the cytoplasm, upstream signaling should enhance formation of the isolation membrane, followed by extension of the membrane by downstream effectors (see overview of autophagy on_Figure 2). When mTOR is inactivated by starvation or inhibitors such as rapamycin, the ULK1 complex facilitates downstream signaling $[31,32]$. Although the true substrate of ULK1 that is involved in induction of autophagy remains to be determined, it is clear that autophagic activity is compromised in the absence of ULK1 kinase; therefore, some target of this kinase must be essential for autophagy [33, 34]. Knocking down ULK1 or its binding partners ATG13 and FIP200 causes a defect in the formation of the isolation membrane observed by conventional electron microscopy (EM), as well as formation of puncta containing downstream autophagy-related proteins $[12,34,35]$. In addition to activating autophagy at an early step, ULK1 also probably plays a role in subsequent steps, as revealed by microscopic observations showing that the ULK1 complex persists for a while even after recruitment of downstream factors. Furthermore, ULK1 is temporarily co-localized with LC3 [36], the best-known autophagosome marker [37] (described below). Although the precise mechanism by which ULK1 facilitates formation of the isolation membrane remains unknown, ULK1-dependent recruitment of the $\mathrm{PI} 3 \mathrm{~K}$ complex is required to produce the isolation membrane, as demonstrated by the observation that the PI3K inhibitor 3-MA and wortmannin strongly inhibit autophagy at an early step [32]. PI3K is a kinase complex that generates phosphatidylinositol phosphates. Among several PI3K complexes, the class III PI3K complex, composed of ATG14, beclin1, VPS15, and VPS34, is essential for autophagy [38-40]. Its relocalization onto autophagosome formation sites results in local production of $\mathrm{PI}$ P at the isolation membrane, which is responsible for recruitment of PI3P-binding downstream components, and also presumably for production of membrane curvature production by Bif-1 [41, 42]. Double FYVE-containing protein 1 (DFCP1, also known as ZFYVE), the ATG18 homolog WIPI1, and two other proteins are recruited to sites of autophagosome formation via their FYVE domains, which are PI3P-binding motifs [43, 44]. The FYVE-containing proteins are believed to facilitate downstream effectors: WIPI2B is required for recruitment of ATG16L complex (LC3 lipidation complex, see below) [45, 46], whereas the role of DFCP1 as an autophagy effector has not yet been determined. It is noteworthy that, in addition to the WIPI2-mediated pathway, proper targeting of ATG16L to sites of autophagosome formation requires direct binding to FIP200, a component of the ULK1 complex, in a manner independent of ATG14, whereas the functional redundancy between those pathways remains unclear [47].

The processing and sequential activation of mammalian ATG8 homologs is a key step in autophagy, as illustrated not only by its essential role in autophagy, but also by the strict and complex mechanism underlying its targeting to isolation membrane. First, the mammalian ATG8 homolog LC3 is processed by Atg4B protease on its C-terminus. The exposure of its C-terminal glycine residue is prerequisite for further modification of LC3 by a sequential reaction similar to ubiquitylation, but in this case LC3 is targeted to phosphatidylethanolamine (PE) [48, 49]. Cleaved LC3 is activated by ATG7 (E1), followed by conjugation with ATG3 (E2) and modification with PE by the ATG12 complex (E3), resulting in formation of LC3-PE (often referred as LC3- Ibecause it can be distinguished from the non-modified form of LC3, which migrates slower in SDS-PAGE) [50]. Most ATG 
proteins involved in LC3 lipidation are essential for autophagy, as demonstrated by the observation that knockout mice lacking these proteins exhibit neonatal lethality; thus, the lipidation process is inevitable for mammalian autophagy in vivo [51]. The role of LC3 itself could not be directly determined in higher eukaryotes because of functional redundancy among three LC3 paralogs in two subfamilies (the LC3 subfamily and GABARAP subfamily). However, significant suppression of autophagic flux by overexpression of the dominant-negative mutant ATG4B (C74A) protein suggests that in mammals, as in yeast, ATG8 is essential for autophagy $[52,53]$. Live-cell imaging with fluorescent tags showed that LC3 forms puncta at sites of autophagosome formation later than upstream components such as ULK1 and WIPI1, suggesting that LC3 plays a role in autophagosome maturation, but not in establishing sites of autophagosome formation. Indeed, atg8-mutated cells exhibit no defects in localization of other autophagy-related proteins on the pre-autophagosomal structure (PAS) in yeast [31]. The proposed roles of LC3 in autophagosome maturation include 1) tethering $[54,55], 2)$ size regulation $[54,55], 3)$ transport $[56,57]$, and 4) sealing of autophagosomes [52, $53,58,59]$. It is possible that these non-mutually exclusive functions are interconnected and form both the autophagosome and the autolysosome (descried in detail below). LC3-PE in the autophagosome is subjected to degradation via autophagy itself, making it powerful marker for measurement autophagic activity, whereas outside the autophagosome, it is recycled by ATG4B-mediated cleavage of PE.

\section{Recipe and topology of autophagosome}

The largest difference between autophagy and other degradation pathways is that autophagy needs a source of membrane to generate organelle. Consequently, there is a topological challenge: how is the autophagic machinery properly positioned to be accessible to the source of membrane? These two important issues, i.e., the membrane 'recipe' of the autophagosome and the topology of autophagosome biogenesis, have been extensively investigated [60]. Previously, two possibilities were considered: membranes could be synthesized de novo or derived from other organelles; however, electron tomographic analysis and live-cell imaging revealed that the endoplasmic reticulum (ER) provides source of autophagosome membranes. Isolation membranes are produced by deformation of the ER membrane and develop into an $\Omega$-shaped structure called the omegasome, followed by extension along the ER membranes; this has been described as the "cradle" model [44, 61, 62]. Additionally, the mitochondrial outer membrane also provides a membrane source for autophagosome formation. This does not contradict to previous findings showing that the autophagosome originates from the ER; instead, recent findings have shown that both are true: simultaneous triple-color live-cell imaging revealed that isolation membranes are formed at contact sites between the ER and mitochondria [63]. In response to starvation signals, components localized onto autophagosome formation sites, such as ATG14 and DFCP1, are enriched in the mitochondria-associated ER membrane (MAM) fraction along with ER and mitochondria proteins. Moreover, among ER-resident SNAREs, STX17 is required for recruitment of the ATG14-containing PI3K complex to ER-mitochondria contact sites, which facilitates autophagy. Knocking down STX17 leads to mislocalization of ATG14, reduced autophagic flux, and a reduction in the number of autolysosomes, suggesting that STX17 plays a role in formation of functional autophagosome. In addition to the ER, the nuclear envelope 
is also considered to be a source of membranes because those two membrane structures are connected with each other. Indeed, the yeast PAS often forms near regions of the nuclear envelope [1].

Although membrane dynamics are essential for autophagy, most ATG proteins lack membrane-binding domains. One exception is ATG9, which has provoked a great deal of interest regarding how this protein facilitates autophagy. A hierarchical analysis of yeast ATG protein recruitment onto the PAS revealed that ATG9 functions upstream of PI3K complex and the ubiquitin-like conjugation system [31], consistent with the observation that deficiency in ATG9 causes a significant defect in LC3 lipidation and autophagy in mammals [64]. Additionally, homozygous deletion of Atg9a results in postnatal lethality in mice, as is the case with Atg5 [65], Atg7 [66], and Atg16L1 [67], confirming the essential physiological role of mammalian ATG9 in autophagy. ATG9 localizes to the Golgi and to distinct compartments called ATG9 vesicles; in yeast, these bodies, which are 30-60 nm in diameter, contain 24-32 ATG molecules each [68, 69]. These observations suggest that ATG9 functions at an early step of isolation membrane formation, although the involvement of ATG9-containing vesicles as a source of autophagosome membrane remains controversial. In mammals, in addition to specific vesicle structures, ATG9 is localized to the Golgi and, in response to starvation stress, is partially enriched in early endosomes [70]. A recent report from S. Tooze's group showed that mAtg9-positive compartments are not integrated into autophagosome, whereas intracellular dynamics of autophagosome rely on an early-acting factor such as Wipi2 [71]. This does not necessarily mean that Atg9 vesicles could not be the source of isolation membrane, but it does imply that the formation process is not simply mediated by fusion between these compartments. It is also possible that ATG9 vesicles retrieve intermediate components such as scaffold proteins (e.g., actin [72])/ lipids for recycling.

Recent finding from R. Schekman's lab added another candidate for the source of autophagosome membranes. They found that the ER-Golgi intermediate compartment (ERGIC), a membrane compartment that facilitates trafficking between the ER and Golgi, has high LC3 lipidation activity [73]. Moreover, functional ERGIC is required for recruitment of DFCP1 and ATG14. A follow-up report showed that ERGIC produces vesicles with LC3 lipidation activity in a manner dependent on PI3K and COPII, supporting the role of ERGIC as a membrane source [74]. The interaction between the ERGIC-dependent pathway and other pathways should be addressed in order to obtain greater mechanistic insight into membrane biogenesis.

\section{Intracellular dynamics of autophagosome for lysosomal digestion}

To prevent massive disintegration of cytoplasmic components by the wide variety of hydrolases contained within the lysosome, the autophagosome must fuse with the lysosome (autolysosome formation) only after the edges of the isolation membrane are sealed. Otherwise, leaked hydrolases would cause cellular damage and apoptosis even at neutral $\mathrm{pH}$ (described below) (Figure 3). As mentioned above, lipidation of LC3 paralogs is essential for autophagosome maturation. In cells overexpressing a dominant-negative form of Atg4B, the numbers of both opened and closed autophagic membranes are elevated. Moreover, Atg5-deficient cells are capable of forming isolation membranes, but not sealing their edges, whereas some upstream components of autophagy are essential for both processes [59]. The 
molecular details underlying the manner in which lipidated LC3 facilitates autophagosome closure have not been fully elucidated; however, LC3 on isolation membranes may recruit other effector proteins through direct binding of their LIRs (LC3-interacting regions) by LC3, an interaction mediated by the highly conserved Lys49 residue [75, 76]. Moreover, because Atg8-PE facilitates membrane hemifusion, LC3-PE itself could promote closure [54]. It is also possible that closure of the autophagosome occurs simultaneously with its fusion with the late endosome/lysosome, i.e., that fusion completes the closure.

Because the autophagosome and lysosome are distinct compartments with characteristic intracellular distributions, their spatiotemporal dynamics are an essential aspect of the process. Lysosomes are primarily located near the centrosome, whereas autophagosomes are generated throughout the cytoplasm. Therefore, the autophagosome has to travel in order to interact with the lysosome. Indeed, the dynamic movements of autophagosomes are significantly upregulated in response to starvation signals in a manner dependent on LC3, as indicated by the inhibition of autophagosome movement by intracellular injection of anti-LC3 antibody [57]. FYCO1 (FYVE and coiled-coil domain-containing protein), a late endosome-enriched protein containing a FYVE domain (binds to PI3P), Rab7 binding domain, and LIR, interacts with LC3 and facilitates autophagosome re-distribution [56]. Deficiency in FYCO1 causes a decrease in autophagic flux and also results in autosomal recessive congenital cataracts, suggesting its role in physiology [56, 77]. Consistent with this idea, lens-specific knockout of Atg5 results in cataracts in mice [78], supporting the function of FYCO1 in autophagy.

The 'head' and 'tail' of autophagy are autophagosome formation and fusion with the lysosome, respectively. As with the head, the tail is also tightly regulated, because it is the point of no return for digestion of cargo. Many unsolved mysteries persist, but recent dramatic advances in the field show that fusion is mediated by multiple mechanisms that could be connected with each other. N. Mizushima's group recently showed that STX17 is the autophagosome SNARE that tethers the autophagosome and lysosome. Tethering is mediated by the interaction between STX17 and SNAP-29, a binding partner of the endosomal/lysosomal SNARE VAMP8 $[79,80]$. This event requires the interaction of STX17 with the HOPS (homotypic fusion and vacuole protein sorting) complex, which facilitates endosome/lysosome fusion [81]. Live-cell imaging showed that STX17 co-localizes with LC3-positive autophagosomes from which other upstream autophagy-related proteins (such as Wipi1) are absent, also suggesting that STX17 functions in a late stage of autophagosome formation. This co-localization also implies that LC3 promotes the fusion event via the STX17/SNAP-29/VAMP8 axis. Indeed, recent work showed that LC3 phosphorylation by Hippo kinase STK3/STK4 is necessary for autophagy downstream of STX17 [82, 83]. Moreover, the HOPS binding protein PLEKHM1 associates with autophagosomes through its LIR domain to promote autolysosome formation [84]. Notably, PLEKHM1 also binds to Rab7, a Rab GTPase localized to late endosomes/lysosomes, whose yeast homolog facilitates homotypic fusion of the vacuole $[85,86]$. Overexpression of dominant-negative Rab7 (T22N) results in inhibition of autolysosome formation [87]; consistent with that, Rab7 is also required for development of large LC3-positive membranes that engulf invading Group A Streptococcus (GAS) [88]. These observations regarding the network centering on LC3 prompted us to propose that LC3 lipidation triggers an integrated system that promotes a series of 
interconnected events: autophagosomal membrane closure, movement, and fusion with the lysosome. This hypothesis is reasonable because these events must be highly coordinated to accomplish the dangerous procedure of fusion, which-like bomb disposal-could wreak havoc if it were to take place in the wrong order.

\section{Negative regulation of autolysosome formation: think before swallowing}

As shown above, autolysosome formation must be highly orchestrated in order to successfully complete fusion between two different organelles. Recent advances in the field revealed the existence of a mechanism to limit this process. Such limitation could be beneficial for cells because fusion represents the point of no return for the whole process; therefore, there should be a checkpoint to determine whether engulfed self components should really be digested, from the standpoints of both nutritional/energetic demand and cellular security. Rubicon (Run domain Beclin-1 interacting and cysteine-rich containing protein) was identified as a negative regulator of UVRAG-PI3K complex, which has been proposed to promote a late step of autophagy [89-91]. Knocking down Rubicon results in accelerated autophagic flux, as shown by elevated autolysosome formation and degradation of autophagic substrates [89-92], indicating that Rubicon acts as a fusion suppressor. Recent studies showed that Rubicon is under tight control by multiple regulatory mechanisms, including mTOR-dependent UVRAG phosphorylation [93], acetylation of beclin-1 [94], and the EGFR-exocyst pathway [95], suggesting that it is the primary gatekeeper and therefore an ideal target for autophagy-related biology. Indeed, Hepatitis virus $C$, which copies itself in the autophagosomes of host cells, manipulates Rubicon to prevent digestion of the virus within the lysosome [96]. Hence, it is important to determine whether Rubicon is involved in a wide range of pathologies, including neurodegeneration and diseases associated with metabolic abnormalities. Moreover, genetic deficiency of Rubicon provides a useful model for study the effect of autophagy induction in vivo, whereas mTOR inhibitors such as rapamycin have potential side effects derived from their broad range of functions [29]; moreover, genetic loss of mTOR function leads to lethality in mice [97]. It should be noted that Rubicon not only control autolysosome formation but also negatively regulates endosome maturation [90, 98] and positively regulates LAP (LC3-associated phagocytosis) in macrophages [99]. Taken together, these observations show that Rubicon plays an important physiological role in autophagy; therefore, it is important to understand the role of Rubicon in vivo. Specifically, it is essential to determine whether Rubicon is involved not only in canonical autophagy but also in selective autophagy, described below.

\section{Selective autophagy as a safeguard against diseases}

To generate energy and building blocks and allow the cell to survive starvation conditions, bulk degradation of cytosolic components does not have to be selective. Electron microscopic studies performed two decades ago showed that autophagic bodies in yeast sometimes contain organelles, such as mitochondria and ribosomes, along with cytoplasmic enzymes [100]; the presence of these components in autophagic bodies could simply reflect their cytoplasmic distribution. Recent advances in autophagy research revealed that cytoplasmic organelles can be specifically targeted by selective autophagy, a process that presumably evolved from non-selective autophagy, which represents the ancestral but 
nonetheless conserved mode. Although conventional non-selective autophagy is certainly essential for human health, selective autophagy plays a more direct role in suppression of a wide variety of diseases. Mitophagy, autophagic digestion of damaged mitochondria, has been extensively investigated because of the vital function of this organelle in cellular homeostasis [101]. To specifically target mitochondria, the autophagic machinery is assembled via interactions with mitochondrial receptor proteins. In yeast, the mitochondrial transmembrane protein Atg32 recruits ATG8 and ATG11, which promote the formation of autophagosomes that engulf mitochondria [102, 103]. Mammalian cells also have several receptor proteins that localize on mitochondria and facilitate mitophagy (discussed more in detail in the following chapter by Yamaguchi et al.). FANDC1 [104], NIX/BNIP3L [105], and BNIP3 [106] contain LIR motifs that interact with LC3, as is the case for Atg32. Interactions between FUNDC1 and LC3 are regulated by phosphorylation and dephosphorylation by CK2 and PGAM5, respectively. Mitophagy is switched on by dephosphorylation of constitutively phosphorylated receptors by CK2, which further promotes assembly of the autophagic machinery [107]. Recently, K. Otsu's group identified Bcl2-L-13 as a functional mammalian homolog of Atg32 [108]. Bcl2-L-13 promotes fragmentation of mitochondria, which is required for engulfment of mitochondria by autophagosomes independently of another important regulatory system for mitophagy, Parkin/PINK1-dependent ubiquitylation. PINK1 kinase is activated by a decrease in mitochondrial membrane potential, followed by phosphorylation of the ubiquitin E3 ligase Parkin, as well as ubiquitin itself, to facilitate ubiquitin-dependent recruitment of autophagic machinery [109-112]. In addition to mitochondria, autophagy can also sequester other organelles, including peroxisomes (pexophagy) [113], the endoplasmic reticulum (ER: ER-phagy) [114, 115], ribosomes (ribophagy), lipid droplets (lipophagy) [116], and lysosomes (lysophagy) [117-119].

\section{A cycle of lysosomal reincarnation; lysophagy maintains lysosomal integrity}

Lysophagy was recently identified as a form of selective autophagy that targets damaged lysosomes (Figure 4). This process is uniquely important for autophagy because the lysosome is essential for digesting contents sequestered by autophagosomes; consequently, a defect in lysophagy could potentially impair autophagy itself. Notably, the role of lysophagy in lysosomal homeostasis in response to lysosome damage, also called lysosomal membrane permeabilization (LMP), is intimately associated with to human pathology. Acute renal failure, a serious type of kidney damage, is often observed after major surgery and chemotherapy [120]. Lysosomal damage stems from crystallization of urate associated with nephropathy; therefore, kidney disease is tightly associated with hyperuricemia [121]. Recent observations showed that dysregulation of renal tubule-specific autophagic deregulation results in exacerbated kidney damage in hyperuricemic model mice treated with uric acid (UA) and the urate oxidase inhibitor oxonic acid (OA) [118]. This observation suggests that lysophagy is a potential candidate for prevention of hyperuricemic nephropathy, and this idea is further supported by the requirement of LC3 for recognition of damaged lysosomes by the autophagic machinery in monosodium urate (MSU)-treated proximal tubular cells. In addition to hyperuricemic nephrophagy, lysophagy could also suppress neurodegeneration and wide range of life-style diseases associated with lysosomal damage, such as type 2 diabetes, gout, and arteriosclerosis [121-127]. Although its direct link to lysophagy has not been 
addressed yet, lysophagy is also expected to be a great target for cardiovascular disease. Danon disease, whose pathology shows cardiomyopathy[128], is caused by hereditable X-linked mutations in a gene encoding LAMP2 which plays inevitable role in lysosome biogenesis as a basic component of lysosomal membrane[129]. Since a deregulated LAMP2 function gives rise to lysosomal membrane damage[130], pathogenesis of the disease might in part stem from LMP, and thus lysophagy might be playing a protective role in the disease. It is also noteworthy that a concurrent lysosomal membrane damage in cardiomyocytes might accumulate with age because of a lipofuscin accumulation inside lysosome predominantly in non-dividing cells[131], suggesting more general role for lysophagy in cardiovascular disease. Furthermore, taken together with the fact that autophagy suppresses cardiovascular disease by maintaining mitochondrial homeostasis and also suppressing inflammation[132-134], it is essential to determine how mitophagy and lysophagy are interconnected to keep cellular homeostasis and control inflammation.

The molecular details of lysophagy have not been fully elucidated, but it is possible that one trigger for lysophagy is exposure of the lysosomal lumen, because recruitment of galectin-3 (a marker of damaged vacuoles) and polyubiquitylation signals on damaged lysosomes are strongly associated with LC3 [117, 118]. This is also the case for recognition of invading bacteria, which disrupt the endosomes that sequester them, followed by labeling of the endosomal lumen with galectin-3 and also galectin-8; the latter not only localizes onto damaged vacuoles but also plays an active role in suppressing bacterial growth inside the cells [135]. This system most likely works as universal pathway for recognizing damaged vacuoles, as indicated by the fact that incorporation of artificial beads rupturing the endosome is sufficient to trigger the same system [136]. Engulfment of damaged lysosomes is followed by fusion with intact lysosomes, resulting in recovery of lysosomal function (Figure 2).

The pathophysiology of lysophagy, which alleviates LMP-associated cytotoxicity and diseases, is also of keen interest in the field. Several lines of evidence suggest that the main function of lysophagy is removal of membrane remnants. Surprisingly, cells seemingly count those membrane remnants as regular lysosomes in maintenance of lysosomal number, as indicated by the fact that the number of lysosomes does not change even after exposure to a sublethal dose of the lysosomotropic reagent LLOMe [118]. This observation implies that a defect in lysophagy leads to prolonged lysosomal malfunction after exposure to lysosomotropic reagents. Moreover, studies of bacterial infection suggest that the damaged membrane itself could exacerbate the inflammatory response [137]. Thus, rebuilding lysosomes from ruptured lysosome is beneficial for human health, because the intracellular "derelict buildings" can potentially damage the cell.

Another challenge in the study of lysophagy is determination of the topology of spikes that break the lysosomal membrane. Some lysosome-damaging agents, such as silica, cannot be processed by enzymatic digestion in lysosomes, raising the question of how they are removed from cells. The answer remains unknown, but it is possible that such materials are somehow stabilized in a non-toxic form inside the cells, or expelled from cells; otherwise, they would cause recurring LMP and cell death regardless of whether lysophagy was functional. Recent studies imply that these materials can be expelled from the cells in an autophagy-dependent manner. Invading bacteria that survive within the lysosome by neutralizing the acidic environment are expelled from the host cells with coats derived from 
autolysosome and exosome membranes [138]. Consistent with this, cytotoxic amyloid beta can be ejected from the cells by the autophagic machinery, leading to formation of the plaques associated with Alzheimer's disease [139]. The autophagic machinery is also hijacked by non-lytic viruses such as poliovirus to escape from host cells in a process known as AWOL (autophagosome-mediated exit without lysis) [140]. Although it remains unknown whether there exists a mechanism that prohibits resorption of exhausted lysosomotropic agents, it is clear that their expulsion reduces their intracellular stay, thereby suppressing cytotoxicity and inflammation.

Lysophagy also sheds new light on iron metabolism. Free heavy ions like iron in lysosomes produce free radicals via Fenton-like reactions, thus facilitating LMP [141]. A recent report showed that ferritin, a chelating protein complex that protects cells from free radical formation by trapping iron ions, is degraded by autophagy through the specific cargo receptor protein NCOA4, a process termed "ferritinophagy" [142, 143]. Because autophagic digestion of ferritin releases free iron into autolysosomes, lysosomes reformed from autolysosome [144-146] presumably contain more free iron. Indeed, lysosomes are rich in free iron, which is heterogeneously distributed among cells and lysosomes [147]. These observations suggest that ferritinophagy promotes lysosome rupture due to the presence of free iron in lysosomes. Consistent with this, cellular sensitivity to hydroxyperoxide is alleviated by NCOA4 knockdown [143], supporting a role for ferritinophagy in lysosomal rupture induced by oxidative stress. Autophagy plays a dual role in lysosome homeostasis, both protective (lysophagy) and sensitizing (ferritinophagy). These influences not only have significant impact on human pathology, as described above, but are also of particular interest to the field of cancer biology because of cancer cells' addiction to autophagy [148, 149] and higher demand for iron [150] for their survival. Therefore, lysophagy-specific regulatory factors represent promising targets for specific elimination of cancer cells by LMP, providing alternative (and presumably better) options for chemotherapy.

\section{Perspectives}

Recent rapid advances in the autophagy field have been accelerated by the involvement of this process in a broad range of physiological phenomena. To establish mechanistic insights, it is essential to investigate unanswered questions regarding the basic mechanisms of autophagy, including the source of autophagosome membrane; the mechanism of isolation membrane extension, closure, and fusion with lysosomes; and the regulatory system that monitors the whole process. The crosstalk among different kinds of selective autophagy is also of keen interest. As evidenced by membrane rupture induced by artificial beads, the recognition system is, at least in part, shared among them and thus they are interconnected each other. Moreover, cells are often exposed to a condition where multiple organelles are damaged at once (e.g., oxidative stress). Degradation of damaged mitochondria by mitophagy can suppress excess ROS, resulting more directly in suppression of LMP. On top of that, as described above, the crosstalk exists not only in cellular autonomous system but also among organs including cardiovascular system. Furthermore, it is important to understand the relationship between autophagy and other cellular signaling cascades, such as the ER stress response, Hippo pathway, DNA damage response, apoptosis, and hypoxia. Those pathways are not only required for their specific signaling cascades, but also expected to suppress generation 
of aggregates degraded by autophagy to maintain homeostasis (e.g. misfolded protein for ER stress). On top of that, they could be directly involved in regulation of autophagy itself. For example, in response to hypoxia associated with energy depletion due to ischemia caused by cardiovascular diseases, a major hypoxic response protein HIF-1 induces autophagy, ER stress response and DNA damage response to coordinate pathways required for tissue survival [151, 152]. These events often happen simultaneously in physiology and interact with one another in vivo; therefore, an integrated understanding of these phenomena is essential for a paradigm shift in autophagy.

\section{Acknowledgements}

We thank the Yoshimori lab members for helpful discussion. We apologize to the many researchers whose excellent articles could not be cited in this manuscript due to page limitations. Work in the authors' laboratory is supported by the Ministry of Education, Culture, Sports, Science and Technology (MEXT) of Japan, and Japan Science and Technology Agency CREST.

\section{References}

[1] Mizushima N, Yoshimori T, Ohsumi Y. The role of Atg proteins in autophagosome formation. Annu Rev Cell Dev Biol. 2011;27:107-32.

[2] Ohsumi Y. Historical landmarks of autophagy research. Cell Res. 2014;24:9-23.

[3] Tsukada M, Ohsumi Y. Isolation and characterization of autophagy-defective mutants of Saccharomyces cerevisiae. FEBS Lett. 1993;333:169-74.

[4] Harding TM, Morano KA, Scott SV, Klionsky DJ. Isolation and characterization of yeast mutants in the cytoplasm to vacuole protein targeting pathway. J Cell Biol. 1995;131:591-602.

[5] Thumm M, Egner R, Koch B, Schlumpberger M, Straub M, Veenhuis M, et al. Isolation of autophagocytosis mutants of Saccharomyces cerevisiae. FEBS Lett. 1994;349:275-80.

[6] Jiang P, Mizushima N. Autophagy and human diseases. Cell Res. 2014;24:69-79.

[7] Cuervo AM, Wong E. Chaperone-mediated autophagy: roles in disease and aging. Cell Res. 2014;24:92-104.

[8] Kaushik S, Bandyopadhyay U, Sridhar S, Kiffin R, Martinez-Vicente M, Kon M, et al. Chaperone-mediated autophagy at a glance. J Cell Sci. 2011;124:495-9.

[9] Efeyan A, Comb WC, Sabatini DM. Nutrient-sensing mechanisms and pathways. Nature. 2015;517:302-10.

[10] Zoncu R, Bar-Peled L, Efeyan A, Wang S, Sancak Y, Sabatini DM. mTORC1 senses lysosomal amino acids through an inside-out mechanism that requires the vacuolar H(+)-ATPase. Science. 2011;334:678-83. 
[11] Sancak Y, Peterson TR, Shaul YD, Lindquist RA, Thoreen CC, Bar-Peled L, et al. The Rag GTPases bind raptor and mediate amino acid signaling to mTORC1. Science. 2008;320:1496-501.

[12] Hosokawa N, Hara T, Kaizuka T, Kishi C, Takamura A, Miura Y, et al. Nutrient-dependent mTORC1 association with the ULK1-Atg13-FIP200 complex required for autophagy. Mol Biol Cell. 2009;20:1981-91.

[13] Hosokawa N, Sasaki T, Iemura S, Natsume T, Hara T, Mizushima N. Atg101, a novel mammalian autophagy protein interacting with Atg13. Autophagy. 2009;5:973-9.

[14] Ganley IG, Lam dH, Wang J, Ding X, Chen S, Jiang X. ULK1.ATG13.FIP200 complex mediates mTOR signaling and is essential for autophagy. J Biol Chem. 2009;284:12297-305.

[15] Jung CH, Jun CB, Ro SH, Kim YM, Otto NM, Cao J, et al. ULK-Atg13-FIP200 complexes mediate mTOR signaling to the autophagy machinery. Mol Biol Cell. 2009;20:1992-2003.

[16] Wong PM, Feng Y, Wang J, Shi R, Jiang X. Regulation of autophagy by coordinated action of mTORC1 and protein phosphatase 2A. Nat Commun. 2015;6:8048.

[17] Holen I, Gordon PB, Seglen PO. Inhibition of hepatocytic autophagy by okadaic acid and other protein phosphatase inhibitors. Eur J Biochem. 1993;215:113-22.

[18] Kim J, Kundu M, Viollet B, Guan KL. AMPK and mTOR regulate autophagy through direct phosphorylation of Ulk1. Nat Cell Biol. 2011;13:132-41.

[19] Egan DF, Shackelford DB, Mihaylova MM, Gelino S, Kohnz RA, Mair W, et al. Phosphorylation of ULK1 (hATG1) by AMP-activated protein kinase connects energy sensing to mitophagy. Science. 2011;331:456-61.

[20] Kamada Y, Funakoshi T, Shintani T, Nagano K, Ohsumi M, Ohsumi Y. Tor-mediated induction of autophagy via an Apg1 protein kinase complex. J Cell Biol. 2000;150:1507-13.

[21] Deter RL, Baudhuin P, De Duve C. Participation of lysosomes in cellular autophagy induced in rat liver by glucagon. J Cell Biol. 1967;35:C11-6.

[22] Seok S, Fu T, Choi SE, Li Y, Zhu R, Kumar S, et al. Transcriptional regulation of autophagy by an FXR-CREB axis. Nature. 2014;516:108-11.

[23] Altarejos JY, Montminy M. CREB and the CRTC co-activators: sensors for hormonal and metabolic signals. Nat Rev Mol Cell Biol. 2011;12:141-51.

[24] Kimball SR, Siegfried BA, Jefferson LS. Glucagon represses signaling through the mammalian target of rapamycin in rat liver by activating AMP-activated protein kinase. J Biol Chem. 2004;279:54103-9.

[25] Baum JI, Kimball SR, Jefferson LS. Glucagon acts in a dominant manner to repress insulin-induced mammalian target of rapamycin complex 1 signaling in perfused rat liver. Am J Physiol Endocrinol Metab. 2009;297:E410-5. 
[26] Fleming A, Noda T, Yoshimori T, Rubinsztein DC. Chemical modulators of autophagy as biological probes and potential therapeutics. Nat Chem Biol. 2011;7:9-17.

[27] Vicinanza M, Korolchuk VI, Ashkenazi A, Puri C, Menzies FM, Clarke JH, et al. PI(5)P regulates autophagosome biogenesis. Mol Cell. 2015;57:219-34.

[28] Williams A, Sarkar S, Cuddon P, Ttofi EK, Saiki S, Siddiqi FH, et al. Novel targets for Huntington's disease in an mTOR-independent autophagy pathway. Nat Chem Biol. 2008;4:295-305.

[29] Laplante M, Sabatini DM. Regulation of mTORC1 and its impact on gene expression at a glance. J Cell Sci. 2013;126:1713-9.

[30] Settembre C, Di Malta C, Polito VA, Garcia Arencibia M, Vetrini F, Erdin S, et al. TFEB links autophagy to lysosomal biogenesis. Science. 2011;332:1429-33.

[31] Suzuki K, Kubota Y, Sekito T, Ohsumi Y. Hierarchy of Atg proteins in pre-autophagosomal structure organization. Genes Cells. 2007;12:209-18.

[32] Itakura E, Mizushima N. Characterization of autophagosome formation site by a hierarchical analysis of mammalian Atg proteins. Autophagy. 2010;6:764-76.

[33] Chan EY, Longatti A, McKnight NC, Tooze SA. Kinase-inactivated ULK proteins inhibit autophagy via their conserved $\mathrm{C}$-terminal domains using an Atg13-independent mechanism. Mol Cell Biol. 2009;29:157-71.

[34] Chan EY, Kir S, Tooze SA. siRNA screening of the kinome identifies ULK1 as a multidomain modulator of autophagy. J Biol Chem. 2007;282:25464-74.

[35] Hara T, Takamura A, Kishi C, Iemura S, Natsume T, Guan JL, et al. FIP200, a ULK-interacting protein, is required for autophagosome formation in mammalian cells. J Cell Biol. 2008;181:497-510.

[36] Karanasios E, Stapleton E, Manifava M, Kaizuka T, Mizushima N, Walker SA, et al. Dynamic association of the ULK1 complex with omegasomes during autophagy induction. J Cell Sci. 2013;126:5224-38.

[37] Kabeya Y, Mizushima N, Ueno T, Yamamoto A, Kirisako T, Noda T, et al. LC3, a mammalian homologue of yeast Apg8p, is localized in autophagosome membranes after processing. EMBO J. 2000;19:5720-8.

[38] Sun Q, Fan W, Chen K, Ding X, Chen S, Zhong Q. Identification of Barkor as a mammalian autophagy-specific factor for Beclin 1 and class III phosphatidylinositol 3-kinase. Proc Natl Acad Sci U S A. 2008;105:19211-6.

[39] Liang XH, Jackson S, Seaman M, Brown K, Kempkes B, Hibshoosh H, et al. Induction of autophagy and inhibition of tumorigenesis by beclin 1 . Nature. 1999;402:672-6.

[40] Kihara A, Kabeya Y, Ohsumi Y, Yoshimori T. Beclin-phosphatidylinositol 3-kinase complex functions at the trans-Golgi network. EMBO Rep. 2001;2:330-5. [41] Takahashi Y, Coppola D, Matsushita N, Cualing HD, Sun M, Sato Y, et al. Bif-1 interacts with Beclin 1 through UVRAG and regulates autophagy and tumorigenesis. Nat Cell Biol. 2007;9:1142-51. 
[42] Takahashi Y, Meyerkord CL, Wang HG. Bif-1/endophilin B1: a candidate for crescent driving force in autophagy. Cell Death Differ. 2009;16:947-55.

[43] Polson HE, de Lartigue J, Rigden DJ, Reedijk M, Urbé S, Clague MJ, et al. Mammalian Atg18 (WIPI2) localizes to omegasome-anchored phagophores and positively regulates LC3 lipidation. Autophagy. 2010;6:506-22.

[44] Axe EL, Walker SA, Manifava M, Chandra P, Roderick HL, Habermann A, et al. Autophagosome formation from membrane compartments enriched in phosphatidylinositol 3-phosphate and dynamically connected to the endoplasmic reticulum. J Cell Biol. 2008;182:685-701.

[45] Dooley HC, Razi M, Polson HE, Girardin SE, Wilson MI, Tooze SA. WIPI2 links LC3 conjugation with PI3P, autophagosome formation, and pathogen clearance by recruiting Atg12-5-16L1. Mol Cell. 2014;55:238-52.

[46] Dooley HC, Wilson MI, Tooze SA. WIPI2B links PtdIns3P to LC3 lipidation through binding ATG16L1. Autophagy. 2015;11:190-1.

[47] Nishimura T, Kaizuka T, Cadwell K, Sahani MH, Saitoh T, Akira S, et al. FIP200 regulates targeting of Atg16L1 to the isolation membrane. EMBO Rep. 2013;14:284-91.

[48] Ichimura Y, Kirisako T, Takao T, Satomi Y, Shimonishi Y, Ishihara N, et al. A ubiquitin-like system mediates protein lipidation. Nature. 2000;408:488-92.

[49] Mizushima N, Noda T, Yoshimori T, Tanaka Y, Ishii T, George MD, et al. A protein conjugation system essential for autophagy. Nature. 1998;395:395-8.

[50] Klionsky DJ, Abdalla FC, Abeliovich H, Abraham RT, Acevedo-Arozena A, Adeli K, et al. Guidelines for the use and interpretation of assays for monitoring autophagy. Autophagy. 2012;8:445-544.

[51] Mizushima N, Levine B. Autophagy in mammalian development and differentiation. Nat Cell Biol. 2010;12:823-30.

[52] Fujita N, Hayashi-Nishino M, Fukumoto H, Omori H, Yamamoto A, Noda T, et al. An Atg4B mutant hampers the lipidation of LC3 paralogues and causes defects in autophagosome closure. Mol Biol Cell. 2008;19:4651-9.

[53] Fujita N, Noda T, Yoshimori T. Atg4B(C74A) hampers autophagosome closure: a useful protein for inhibiting autophagy. Autophagy. 2009;5:88-9.

[54] Nakatogawa H, Ichimura Y, Ohsumi Y. Atg8, a ubiquitin-like protein required for autophagosome formation, mediates membrane tethering and hemifusion. Cell. 2007;130:165-78.

[55] Xie Z, Nair U, Klionsky DJ. Atg8 controls phagophore expansion during autophagosome formation. Mol Biol Cell. 2008;19:3290-8.

[56] Pankiv S, Alemu EA, Brech A, Bruun JA, Lamark T, Overvatn A, et al. FYCO1 is a Rab7 effector that binds to LC3 and PI3P to mediate microtubule plus end-directed vesicle transport. J Cell Biol. 2010;188:253-69.

[57] Kimura S, Noda T, Yoshimori T. Dynein-dependent movement of autophagosomes mediates efficient encounters with lysosomes. Cell Struct Funct. 2008;33:109-22. 
[58] Weidberg H, Shvets E, Shpilka T, Shimron F, Shinder V, Elazar Z. LC3 and GATE-16/GABARAP subfamilies are both essential yet act differently in autophagosome biogenesis. EMBO J. 2010;29:1792-802.

[59] Kishi-Itakura C, Koyama-Honda I, Itakura E, Mizushima N. Ultrastructural analysis of autophagosome organization using mammalian autophagy-deficient cells. J Cell Sci. 2014;127:4089-102.

[60] Tooze SA, Yoshimori T. The origin of the autophagosomal membrane. Nat Cell Biol. 2010;12:831-5.

[61] Hayashi-Nishino M, Fujita N, Noda T, Yamaguchi A, Yoshimori T, Yamamoto A. A subdomain of the endoplasmic reticulum forms a cradle for autophagosome formation. Nat Cell Biol. 2009;11:1433-7.

[62] Hayashi-Nishino M, Fujita N, Noda T, Yamaguchi A, Yoshimori T, Yamamoto A. Electron tomography reveals the endoplasmic reticulum as a membrane source for autophagosome formation. Autophagy. 2010;6:301-3.

[63] Hamasaki M, Furuta N, Matsuda A, Nezu A, Yamamoto A, Fujita N, et al. Autophagosomes form at ER-mitochondria contact sites. Nature. 2013;495:389-93.

[64] Saitoh T, Fujita N, Yoshimori T, Akira S. Regulation of dsDNA-induced innate immune responses by membrane trafficking. Autophagy. 2010;6:430-2.

[65] Kuma A, Hatano M, Matsui M, Yamamoto A, Nakaya H, Yoshimori T, et al. The role of autophagy during the early neonatal starvation period. Nature. 2004;432:1032-6.

[66] Komatsu M, Waguri S, Ueno T, Iwata J, Murata S, Tanida I, et al. Impairment of starvation-induced and constitutive autophagy in Atg7-deficient mice. J Cell Biol. 2005;169:425-34.

[67] Saitoh T, Fujita N, Jang MH, Uematsu S, Yang BG, Satoh T, et al. Loss of the autophagy protein Atg16L1 enhances endotoxin-induced IL-1beta production. Nature. 2008;456:264-8.

[68] Yamamoto H, Kakuta S, Watanabe TM, Kitamura A, Sekito T, Kondo-Kakuta C, et al. Atg9 vesicles are an important membrane source during early steps of autophagosome formation. J Cell Biol. 2012;198:219-33.

[69] Mari M, Griffith J, Rieter E, Krishnappa L, Klionsky DJ, Reggiori F. An Atg9-containing compartment that functions in the early steps of autophagosome biogenesis. J Cell Biol. 2010;190:1005-22.

[70] Young AR, Chan EY, Hu XW, Köchl R, Crawshaw SG, High S, et al. Starvation and ULK1-dependent cycling of mammalian Atg9 between the TGN and endosomes. J Cell Sci. 2006;119:3888-900.

[71] Orsi A, Razi M, Dooley HC, Robinson D, Weston AE, Collinson LM, et al. Dynamic and transient interactions of Atg9 with autophagosomes, but not membrane integration, are required for autophagy. Mol Biol Cell. 2012;23:1860-73. 
[72] Mi N, Chen Y, Wang S, Chen M, Zhao M, Yang G, et al. CapZ regulates autophagosomal membrane shaping by promoting actin assembly inside the isolation membrane. Nat Cell Biol. 2015.

[73] Ge L, Melville D, Zhang M, Schekman R. The ER-Golgi intermediate compartment is a key membrane source for the LC3 lipidation step of autophagosome biogenesis. Elife. 2013;2:e00947.

[74] Ge L, Zhang M, Schekman R. Phosphatidylinositol 3-kinase and COPII generate LC3 lipidation vesicles from the ER-Golgi intermediate compartment. Elife. 2014;3:e04135.

[75] Suzuki H, Tabata K, Morita E, Kawasaki M, Kato R, Dobson RC, et al. Structural basis of the autophagy-related LC3/Atg13 LIR complex: recognition and interaction mechanism. Structure. 2014;22:47-58.

[76] Kalvari I, Tsompanis S, Mulakkal NC, Osgood R, Johansen T, Nezis IP, et al. iLIR: A web resource for prediction of Atg8-family interacting proteins. Autophagy. 2014;10:913-25.

[77] Chen J, Ma Z, Jiao X, Fariss R, Kantorow WL, Kantorow M, et al. Mutations in FYCO1 cause autosomal-recessive congenital cataracts. Am J Hum Genet. 2011;88:827-38.

[78] Morishita H, Eguchi S, Kimura H, Sasaki J, Sakamaki Y, Robinson ML, et al. Deletion of autophagy-related 5 (Atg5) and Pik3c3 genes in the lens causes cataract independent of programmed organelle degradation. J Biol Chem. 2013;288:11436-47.

[79] Itakura E, Kishi-Itakura C, Mizushima N. The hairpin-type tail-anchored SNARE syntaxin 17 targets to autophagosomes for fusion with endosomes/lysosomes. Cell. 2012;151:1256-69.

[80] Itakura E, Mizushima N. Syntaxin 17: the autophagosomal SNARE. Autophagy. 2013;9:917-9.

[81] Jiang P, Nishimura T, Sakamaki Y, Itakura E, Hatta T, Natsume T, et al. The HOPS complex mediates autophagosome-lysosome fusion through interaction with syntaxin 17. Mol Biol Cell. 2014;25:1327-37.

[82] Wilkinson DS, Hansen M. LC3 is a novel substrate for the mammalian Hippo kinases, STK3/STK4. Autophagy. 2015;11:856-7.

[83] Wilkinson DS, Jariwala JS, Anderson E, Mitra K, Meisenhelder J, Chang JT, et al. Phosphorylation of LC3 by the Hippo kinases STK3/STK4 is essential for autophagy. Mol Cell. 2015;57:55-68.

[84] McEwan DG, Popovic D, Gubas A, Terawaki S, Suzuki H, Stadel D, et al. PLEKHM1 regulates autophagosome-lysosome fusion through HOPS complex and LC3/GABARAP proteins. Mol Cell. 2015;57:39-54.

[85] Tabata K, Matsunaga K, Sakane A, Sasaki T, Noda T, Yoshimori T. Rubicon and PLEKHM1 negatively regulate the endocytic/autophagic pathway via a novel Rab7-binding domain. Mol Biol Cell. 2010;21:4162-72. 
[86] Haas A, Scheglmann D, Lazar T, Gallwitz D, Wickner W. The GTPase Ypt7p of Saccharomyces cerevisiae is required on both partner vacuoles for the homotypic fusion step of vacuole inheritance. EMBO J. 1995;14:5258-70.

[87] Kimura S, Noda T, Yoshimori T. Dissection of the autophagosome maturation process by a novel reporter protein, tandem fluorescent-tagged LC3. Autophagy. 2007;3:452-60.

[88] Yamaguchi H, Nakagawa I, Yamamoto A, Amano A, Noda T, Yoshimori T. An initial step of GAS-containing autophagosome-like vacuoles formation requires Rab7. PLoS Pathog. 2009;5:e1000670.

[89] Matsunaga K, Noda T, Yoshimori T. Binding Rubicon to cross the Rubicon. Autophagy. 2009;5:876-7.

[90] Matsunaga K, Saitoh T, Tabata K, Omori H, Satoh T, Kurotori N, et al. Two Beclin 1-binding proteins, Atg14L and Rubicon, reciprocally regulate autophagy at different stages. Nat Cell Biol. 2009;11:385-96.

[91] Zhong Y, Wang QJ, Li X, Yan Y, Backer JM, Chait BT, et al. Distinct regulation of autophagic activity by Atg14L and Rubicon associated with Beclin 1-phosphatidylinositol-3-kinase complex. Nat Cell Biol. 2009;11:468-76.

[92] Sun Q, Zhang J, Fan W, Wong KN, Ding X, Chen S, et al. The RUN domain of rubicon is important for hVps34 binding, lipid kinase inhibition, and autophagy suppression. J Biol Chem. 2011;286:185-91.

[93] Kim YM, Jung CH, Seo M, Kim EK, Park JM, Bae SS, et al. mTORC1 phosphorylates UVRAG to negatively regulate autophagosome and endosome maturation. Mol Cell. 2015;57:207-18.

[94] Sun T, Li X, Zhang P, Chen WD, Zhang HL, Li DD, et al. Acetylation of Beclin 1 inhibits autophagosome maturation and promotes tumour growth. Nat Commun. 2015;6:7215.

[95] Tan X, Thapa N, Sun Y, Anderson RA. A kinase-independent role for EGF receptor in autophagy initiation. Cell. 2015;160:145-60.

[96] Wang L, Tian Y, Ou JH. HCV induces the expression of Rubicon and UVRAG to temporally regulate the maturation of autophagosomes and viral replication. PLoS Pathog. 2015;11:e1004764.

[97] Murakami M, Ichisaka T, Maeda M, Oshiro N, Hara K, Edenhofer F, et al. mTOR is essential for growth and proliferation in early mouse embryos and embryonic stem cells. Mol Cell Biol. 2004;24:6710-8.

[98] Sun Q, Westphal W, Wong KN, Tan I, Zhong Q. Rubicon controls endosome maturation as a Rab7 effector. Proc Natl Acad Sci U S A. 2010;107:19338-43.

[99] Martinez J, Malireddi RK, Lu Q, Cunha LD, Pelletier S, Gingras S, et al. Molecular characterization of LC3-associated phagocytosis reveals distinct roles for Rubicon, NOX2 and autophagy proteins. Nat Cell Biol. 2015;17:893-906.

[100] Baba M, Takeshige K, Baba N, Ohsumi Y. Ultrastructural analysis of the autophagic process in yeast: detection of autophagosomes and their characterization. J Cell Biol. 1994;124:903-13. 
[101] Liu L, Sakakibara K, Chen Q, Okamoto K. Receptor-mediated mitophagy in yeast and mammalian systems. Cell Res. 2014;24:787-95.

[102] Okamoto K, Kondo-Okamoto N, Ohsumi Y. Mitochondria-anchored receptor Atg32 mediates degradation of mitochondria via selective autophagy. Dev Cell. 2009;17:87-97.

[103] Kanki T, Wang K, Cao Y, Baba M, Klionsky DJ. Atg32 is a mitochondrial protein that confers selectivity during mitophagy. Dev Cell. 2009;17:98-109.

[104] Liu L, Feng D, Chen G, Chen M, Zheng Q, Song P, et al. Mitochondrial outer-membrane protein FUNDC1 mediates hypoxia-induced mitophagy in mammalian cells. Nat Cell Biol. 2012;14:177-85.

[105] Novak I, Kirkin V, McEwan DG, Zhang J, Wild P, Rozenknop A, et al. Nix is a selective autophagy receptor for mitochondrial clearance. EMBO Rep. 2010;11:45-51.

[106] Hanna RA, Quinsay MN, Orogo AM, Giang K, Rikka S, Gustafsson A. Microtubule-associated protein 1 light chain 3 (LC3) interacts with Bnip3 protein to selectively remove endoplasmic reticulum and mitochondria via autophagy. J Biol Chem. 2012;287:19094-104.

[107] Chen G, Han Z, Feng D, Chen Y, Chen L, Wu H, et al. A regulatory signaling loop comprising the PGAM5 phosphatase and CK2 controls receptor-mediated mitophagy. Mol Cell. 2014;54:362-77.

[108] Murakawa T, Yamaguchi O, Hashimoto A, Hikoso S, Takeda T, Oka T, et al. Bcl-2-like protein 13 is a mammalian Atg32 homologue that mediates mitophagy and mitochondrial fragmentation. Nat Commun. 2015;6:7527.

[109] Lazarou M, Sliter DA, Kane LA, Sarraf SA, Wang C, Burman JL, et al. The ubiquitin kinase PINK1 recruits autophagy receptors to induce mitophagy. Nature. 2015.

[110] Koyano F, Okatsu K, Kosako H, Tamura Y, Go E, Kimura M, et al. Ubiquitin is phosphorylated by PINK1 to activate parkin. Nature. 2014;510:162-6.

[111] Kondapalli C, Kazlauskaite A, Zhang N, Woodroof HI, Campbell DG, Gourlay R, et al. PINK1 is activated by mitochondrial membrane potential depolarization and stimulates Parkin E3 ligase activity by phosphorylating Serine 65. Open Biol. 2012;2:120080.

[112] Shiba-Fukushima K, Imai Y, Yoshida S, Ishihama Y, Kanao T, Sato S, et al. PINK1-mediated phosphorylation of the Parkin ubiquitin-like domain primes mitochondrial translocation of Parkin and regulates mitophagy. Sci Rep. 2012;2:1002.

[113] Manjithaya R, Nazarko TY, Farré JC, Subramani S. Molecular mechanism and physiological role of pexophagy. FEBS Lett. 2010;584:1367-73.

[114] Khaminets A, Heinrich T, Mari M, Grumati P, Huebner AK, Akutsu M, et al. Regulation of endoplasmic reticulum turnover by selective autophagy. Nature. $2015 ; 522: 354-8$. 
[115] Mochida K, Oikawa Y, Kimura Y, Kirisako H, Hirano H, Ohsumi Y, et al. Receptor-mediated selective autophagy degrades the endoplasmic reticulum and the nucleus. Nature. 2015;522:359-62.

[116] Singh R, Kaushik S, Wang Y, Xiang Y, Novak I, Komatsu M, et al. Autophagy regulates lipid metabolism. Nature. 2009;458:1131-5.

[117] Hung YH, Chen LM, Yang JY, Yang WY. Spatiotemporally controlled induction of autophagy-mediated lysosome turnover. Nat Commun. 2013;4:2111.

[118] Maejima I, Takahashi A, Omori H, Kimura T, Takabatake Y, Saitoh T, et al. Autophagy sequesters damaged lysosomes to control lysosomal biogenesis and kidney injury. EMBO J. 2013;32:2336-47.

[119] Hasegawa J, Maejima I, Iwamoto R, Yoshimori T. Selective autophagy: lysophagy. Methods. 2015;75:128-32.

[120] Ejaz AA, Mu W, Kang DH, Roncal C, Sautin YY, Henderson G, et al. Could uric acid have a role in acute renal failure? Clin J Am Soc Nephrol. 2007;2:16-21.

[121] Isaka Y, Takabatake Y, Takahashi A, Saitoh T, Yoshimori T. Hyperuricemia-induced inflammasome and kidney diseases. Nephrol Dial Transplant. 2015.

[122] Rivera JF, Costes S, Gurlo T, Glabe CG, Butler PC. Autophagy defends pancreatic $\beta$ cells from human islet amyloid polypeptide-induced toxicity. J Clin Invest. 2014;124:3489-500.

[123] Shigihara N, Fukunaka A, Hara A, Komiya K, Honda A, Uchida T, et al. Human IAPP-induced pancreatic $\beta$ cell toxicity and its regulation by autophagy. $J$ Clin Invest. 2014; $124: 3634-44$.

[124] Kim J, Cheon H, Jeong YT, Quan W, Kim KH, Cho JM, et al. Amyloidogenic peptide oligomer accumulation in autophagy-deficient $\beta$ cells induces diabetes. J Clin Invest. 2014;124:3311-24.

[125] Dehay B, Bové J, Rodríguez-Muela N, Perier C, Recasens A, Boya P, et al. Pathogenic lysosomal depletion in Parkinson's disease. J Neurosci. 2010;30:12535-44.

[126] Freeman D, Cedillos R, Choyke S, Lukic Z, McGuire K, Marvin S, et al. Alpha-synuclein induces lysosomal rupture and cathepsin dependent reactive oxygen species following endocytosis. PLoS One. 2013;8:e62143.

[127] Duewell P, Kono H, Rayner KJ, Sirois CM, Vladimer G, Bauernfeind FG, et al. NLRP3 inflammasomes are required for atherogenesis and activated by cholesterol crystals. Nature. 2010;464:1357-61.

[128] D'souza RS, Levandowski C, Slavov D, Graw SL, Allen LA, Adler E, et al. Danon disease: clinical features, evaluation, and management. Circ Heart Fail. 2014;7:843-9.

[129] Nishino I, Fu J, Tanji K, Yamada T, Shimojo S, Koori T, et al. Primary LAMP-2 deficiency causes X-linked vacuolar cardiomyopathy and myopathy (Danon disease). Nature. 2000;406:906-10. 
[130] Fehrenbacher N, Bastholm L, Kirkegaard-Sørensen T, Rafn B, Bøttzauw T, Nielsen C, et al. Sensitization to the lysosomal cell death pathway by oncogene-induced down-regulation of lysosome-associated membrane proteins 1 and 2. Cancer Res. 2008;68:6623-33.

[131] Brunk UT, Terman A. Lipofuscin: mechanisms of age-related accumulation and influence on cell function. Free Radic Biol Med. 2002;33:611-9.

[132] Oka T, Hikoso S, Yamaguchi O, Taneike M, Takeda T, Tamai T, et al. Mitochondrial DNA that escapes from autophagy causes inflammation and heart failure. Nature. 2012;485:251-5.

[133] Taneike M, Yamaguchi O, Nakai A, Hikoso S, Takeda T, Mizote I, et al. Inhibition of autophagy in the heart induces age-related cardiomyopathy. Autophagy. 2010;6:600-6.

[134] Nakai A, Yamaguchi O, Takeda T, Higuchi Y, Hikoso S, Taniike M, et al. The role of autophagy in cardiomyocytes in the basal state and in response to hemodynamic stress. Nat Med. 2007;13:619-24.

[135] Thurston TL, Wandel MP, von Muhlinen N, Foeglein A, Randow F. Galectin 8 targets damaged vesicles for autophagy to defend cells against bacterial invasion. Nature. 2012;482:414-8.

[136] Fujita N, Morita E, Itoh T, Tanaka A, Nakaoka M, Osada Y, et al. Recruitment of the autophagic machinery to endosomes during infection is mediated by ubiquitin. J Cell Biol. 2013;203:115-28.

[137] Dupont N, Lacas-Gervais S, Bertout J, Paz I, Freche B, Van Nhieu GT, et al. Shigella phagocytic vacuolar membrane remnants participate in the cellular response to pathogen invasion and are regulated by autophagy. Cell Host Microbe. 2009;6:137-49.

[138] Miao Y, Li G, Zhang X, Xu H, Abraham SN. A TRP Channel Senses Lysosome Neutralization by Pathogens to Trigger Their Expulsion. Cell. 2015;161:1306-19.

[139] Nilsson P, Loganathan K, Sekiguchi M, Matsuba Y, Hui K, Tsubuki S, et al. A secretion and plaque formation depend on autophagy. Cell Rep. 2013;5:61-9.

[140] Bird SW, Kirkegaard K. Escape of non-enveloped virus from intact cells. Virology. 2015;479-480:444-9.

[141] Kurz T, Eaton JW, Brunk UT. The role of lysosomes in iron metabolism and recycling. Int J Biochem Cell Biol. 2011;43:1686-97.

[142] Goodall M, Thorburn A. Identifying specific receptors for cargo-mediated autophagy. Cell Res. 2014;24:783-4.

[143] Mancias JD, Wang X, Gygi SP, Harper JW, Kimmelman AC. Quantitative proteomics identifies NCOA4 as the cargo receptor mediating ferritinophagy. Nature. 2014;509:105-9.

[144] Yu L, McPhee CK, Zheng L, Mardones GA, Rong Y, Peng J, et al. Termination of autophagy and reformation of lysosomes regulated by mTOR. Nature. 2010;465:942-6. 
[145] Rong Y, McPhee CK, McPhee C, Deng S, Huang L, Chen L, et al. Spinster is required for autophagic lysosome reformation and mTOR reactivation following starvation. Proc Natl Acad Sci U S A. 2011;108:7826-31.

[146] Rong Y, Liu M, Ma L, Du W, Zhang H, Tian Y, et al. Clathrin and phosphatidylinositol-4,5-bisphosphate regulate autophagic lysosome reformation. Nat Cell Biol. 2012;14:924-34.

[147] Kurz T, Gustafsson B, Brunk UT. Cell sensitivity to oxidative stress is influenced by ferritin autophagy. Free Radic Biol Med. 2011;50:1647-58.

[148] Maes H, Rubio N, Garg AD, Agostinis P. Autophagy: shaping the tumor microenvironment and therapeutic response. Trends Mol Med. 2013;19:428-46.

[149] Kimura T, Takabatake Y, Takahashi A, Isaka Y. Chloroquine in cancer therapy: a double-edged sword of autophagy. Cancer Res. 2013;73:3-7.

[150] Torti SV, Torti FM. Cellular iron metabolism in prognosis and therapy of breast cancer. Crit Rev Oncog. 2013;18:435-48.

[151] Mazure NM, Pouysségur J. Hypoxia-induced autophagy: cell death or cell survival? Curr Opin Cell Biol. 2010;22:177-80.

[152] Xu C, Bailly-Maitre B, Reed JC. Endoplasmic reticulum stress: cell life and death decisions. J Clin Invest. 2005;115:2656-64. 


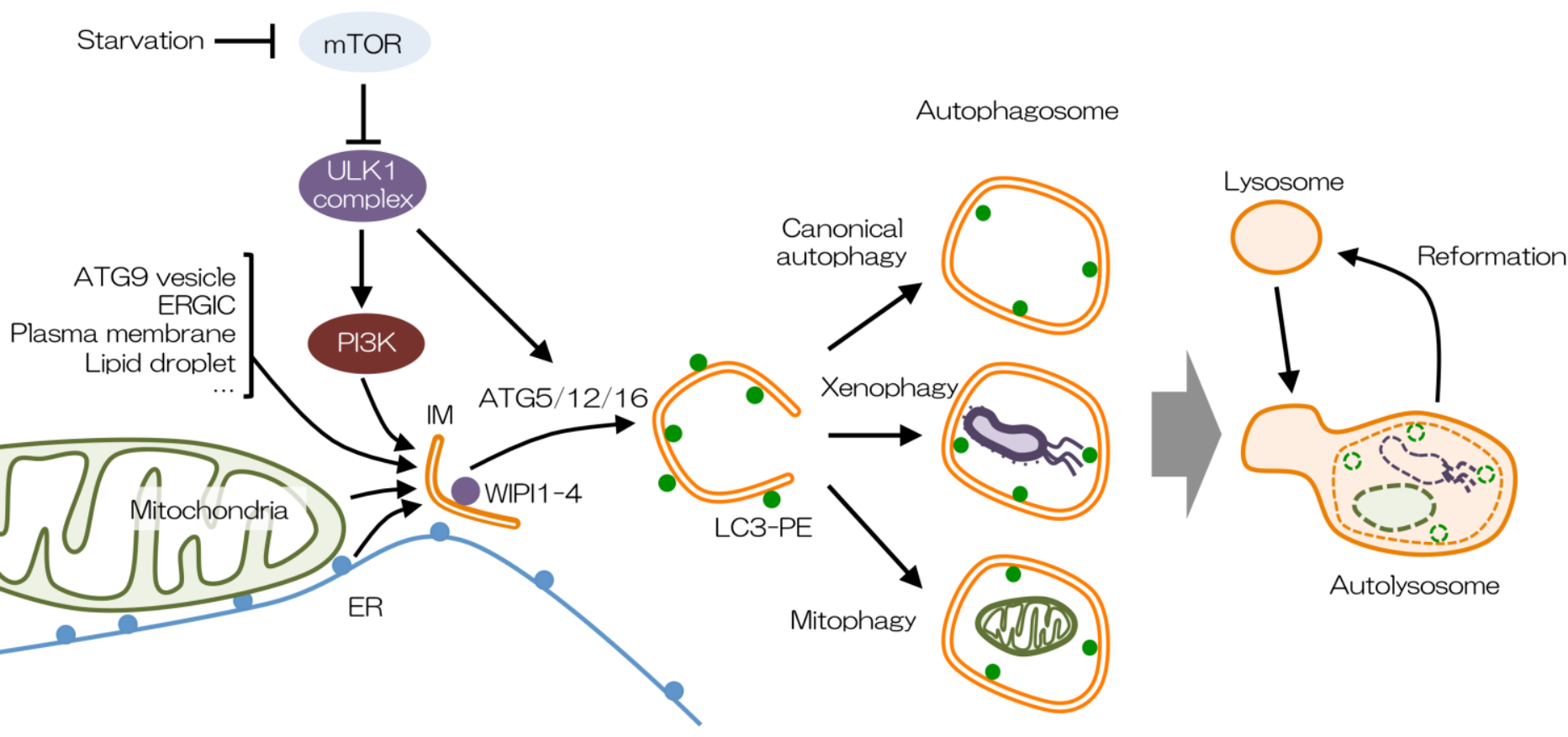

Figure 2. An overview of mammalian autophagosome/autolysosome biogenesis. Nutrient deprivation unlocks the ULK1 complex inhibited by mTOR-dependent phosphorylation. An active ULK1 complex is responsible to recruit PI3K (ATG14/Beclin1/VPS34/VPS15) and also downstream effectors to facilitate formation of isolation membrane (IM) at mitochondria-ER contact site. As other source of autophagosome, ERGIC, plasma membrane, lipid droplets, and ATG9 vesicles support IM formation and elongation. PI3P binding proteins such as WIPI2 recruit LC3-conjugation system, promoting autophagosome closure via LC3 paralogs. Autophagosomes are further fused with lysosome, followed by enzymatic digestion of the inner components. After persistent starvation, lysosome can be re-established from autolysosome (ALR: Autophagic Lysosome Reformation). Not only obtaining building blocks and energy for starvation, autophagy also works as safeguard system against invading bacteria (Xenophagy), compromised organelles such as mitochondria (mitophagy) and even also lysosome itself (lysophagy, described later in detail). 


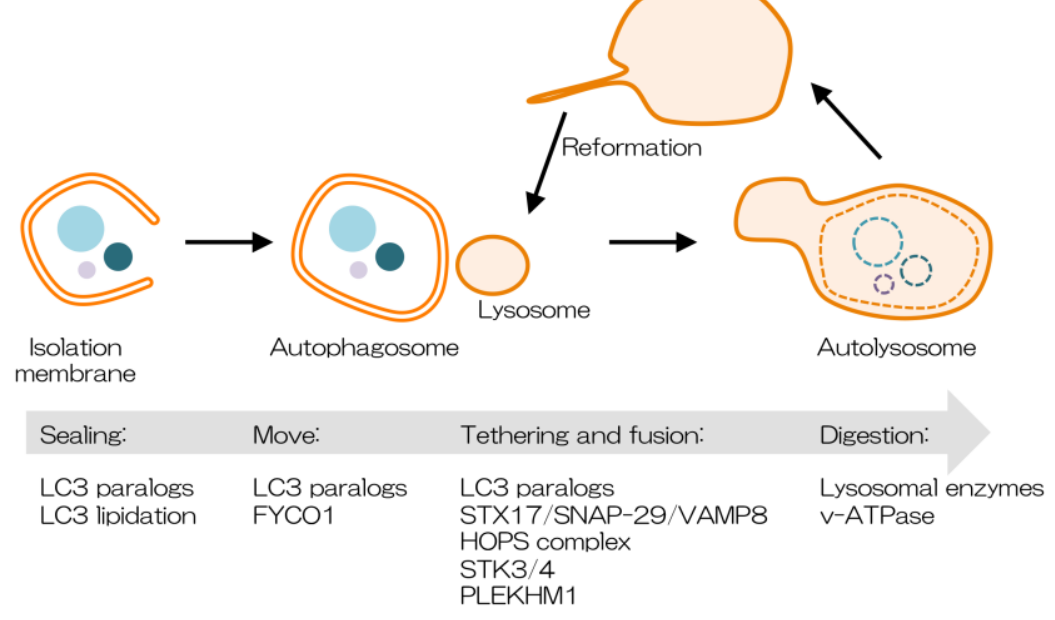

Figure 3. Sequential progression of autolysosome formation . To

achieve faithful completion of autophagy, it is inevitable to promote a series of actions in a highly orchestrated manner. LC3 palalogs and their lipidation are required throughout the process, suggesting their role as master regulator of autolysosome formation. An intracellular dynamics of autophagosome is needed to fuse with lysosome, facilitated by FYCO-mediated loading of autophagosome onto a dynein motor via its binding to LC3 on autophagsome. A tethering autophagosome with lysosome is promoted by autophagosomal SNARE protein Syntaxin 17 and lysosomal SNARE VAMP8 which are connected each other by SNAP-29. Additionally, this process is mediated by HOPS proteins. LC3 paralogs are also required for the fusion event via phosphorylation by STK3/4 and binding to PLEKHM1. To digest a cargo by lysosomal enzymes in autolysosome, v-ATPase maintains low-pH suitable for them. Used autolysosome can be utilized for reformation of lysososme. Note that the fusion process is controlled by negative regulator Rubicon, which is further regulated by several pathways. 


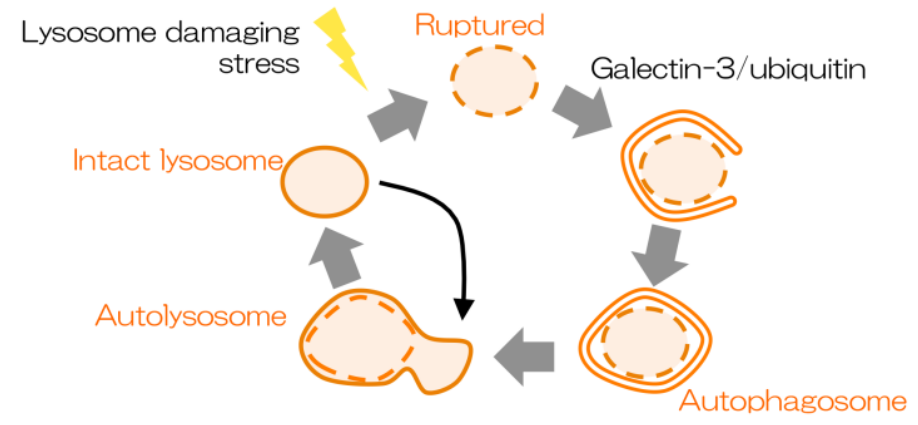

Figure 4. Lysophagy facilitates lysosomal function utilizing intact lysosome itself. Lysosomal membrane permeabilization is induced by environmental stress such as oxidative stress and an accumulation of urate crystal, but also lysosome damaging reagents. Ruptured lysosome is coated with endogeneous galectin-3 protein, followed by ubiquitylation. An autophagy machinery promotes engulfment of ubiquitin positive ruptured lysosome in a manner similar to other selective autophagy, then fused with intact lysosome to recover functional lysosome. 